\title{
Particle Dynamics Around a Charged Black Hole
}

\author{
Sehrish Iftikhar $^{1, \star}$ \\ ${ }^{1}$ Department of Mathematics, \\ Lahore College for Women University, Lahore-54000, Pakistan.
}

\begin{abstract}
This paper investigates particle dynamics around the noncommutative Reissner Nordström black hole. We study escape velocity of the particle at innermost stable circular orbit. In order to discuss the stability of orbits we analyze effective potential. We compare our results with the dynamics of particles in Reissner Nordström as well as noncommutative Schwarzschild black hole. We observe that the noncommutative parameter affects the motion of particles.
\end{abstract}

\section{Introduction}

It is believed that the concept of continuous spacetime breaks down at short distances (of the order of Planck lenght). The noncommutative (NC) theory proved to be very useful to discuss the dynamics of spacetime at Planck scale. The NC geometry is described by universal minimal length scale, $\sqrt{\Theta}$ where, $\Theta$ is a deformation parameter that is equivalent to Planck's length. The noncommutativity of spacetime is based on quantum mechanics. In this regard, the NC spacetime is described by Hermitian operators that are not commutative, i.e., [1]

$$
\left[x^{\alpha}, x^{\beta}\right]=\iota \Theta^{\alpha \beta},
$$

where $\Theta^{\alpha \beta}$ is antisymmetric matrix that has dimension (lenght) ${ }^{2}$, also it is real valued. It describes a small region in $\alpha \beta$-plane (of the observable domain) as the Planck's constant represents very small fundamental cell of the observable phase space in quantum mechanics. The ordinary spacetime can be obtained in the limit $\Theta \rightarrow 0$.

The existence of black holes (BHs) is one of the most prominent predictions of general relativity (GR). Black holes are the regions in space in which singularities are hidden inside the event horizons. Generally, in the framework of quantum mechanics, the singular sources are smeared out and the spacetime is described by NC geometry. Such modifications to GR have a compelling effect on the $\mathrm{BH}$ structure. Many other interesting features of NC spacetime have been studied in literature [2].

The study of geodesics (null or timelike) explains the properties related to the geometry of curved spacetime. Among the different types of geodesics, circular geodesics are of great importance for astrophysical as well as theoretical research. Motion of particles in weakly magnetized Schwarzschild BH was considered by Frolov and shoom [3]. They mainly focused on the bounded trajectories in equatorial plane. Frolov [4] explored the collision of particles for the Schwarzschild BH and found

^e-mail: sehrish3iftikhar@gmail.com 
that in the strong magnetic field, maximal collision energy can be high. Zahrani et al. [5] studied the escape velocity for particles at the innermost stable circular orbit (ISCO) and found that such motion is chaotic, generally. Some interesting features of neutral as well as charged particle dynamics are discussed in [6]

In this paper, we explore the dynamics of particles around Noncommutative Reissner Nordström (NCRN) BH. The paper is organized as follows. In section 2, we present the NCRN BH and study the equations of motion. In section $\mathbf{3}$ we numerically analyze the behavior of effective potential as well as escape velocity of the particle. The results are summarized in the last section.

\section{Equations of Motion}

In order to discuss the $\mathrm{BHs}$ in the context of $\mathrm{NC}$ geometry, the corresponding field equations have to be solved. In this regard, the Einstein tensor remains unchanged and the effect of noncommutativity is taken into account only on the matter part (energy momentum tensor) [7-9]. This approach is analogues to the NC modification of quantum field theory [10]. The effect of smearing is carried out by considering energy density of a static, spherically symmetric, particle-like, smeared gravitational source [7-9]

$$
\rho_{\Theta}=\frac{M}{(4 \pi \Theta)^{\frac{3}{2}}} e^{-\frac{r^{2}}{4 \Theta}}
$$

where $M$ denotes the mass of the particle diffused throughout the region of linear size $\sqrt{\Theta}$ and $\Theta$ represents the NC parameter. The spherically symmetric line element by solving the Einstein field equations with $\rho_{\Theta}$, is given by [11]

$$
d s^{2}=-h(r) d t^{2}+h(r)^{-1} d r^{2}+r^{2}\left(d \theta^{2}+\sin ^{2} \theta d \phi^{2}\right),
$$

where

$$
h(r)=1-\frac{2 M_{\Theta}}{r}+\frac{Q_{\Theta}^{2}}{r^{2}}
$$

here

$$
\begin{gathered}
M_{\Theta}=\frac{2 M}{\sqrt{\pi}} \gamma\left(\frac{3}{2}, \frac{r^{2}}{4 \Theta}\right), \\
Q_{\Theta}=\frac{Q}{\sqrt{\pi}} \sqrt{\gamma^{2}\left(\frac{1}{2}, \frac{r^{2}}{4 \Theta}\right)-\frac{r}{\sqrt{2 \Theta}} \gamma\left(\frac{1}{2}, \frac{r^{2}}{2 \Theta}\right),}
\end{gathered}
$$

and

$$
\gamma\left(\frac{a}{b}, x\right)=\int_{0}^{x} \frac{d \mu}{\mu} \mu^{\frac{a}{b}} e^{-\mu}
$$

is the incomplete gamma function and $Q$ is the charge. In the limit $\frac{r}{\sqrt{\Theta}} \rightarrow \infty$, the classical RN BH is recovered.

Using $f(r)=0$, we find the horizons

$$
r_{h}=\frac{M_{\Theta} \pm \sqrt{M_{\Theta}^{2}-4 Q_{\Theta}^{2}}}{2},
$$

This leads to a complicated equation, therefore it is difficult to find the horizons in closed form. A detailed discussion about horizons and other important properties for spacetime (2) is given in [11]. For a spherically symmetric spacetime, there are three integrals of motion. Two of them are related to 
the symmetries of the $\mathrm{BH}$ metric with respect to translations of time and rotations about the symmetry axis and are obtained by the Killing vectors, given by

$$
\xi_{(t)}^{v} \partial_{v}=\partial_{t}, \quad \xi_{(\phi)}^{v} \partial_{v}=\partial_{\phi}
$$

here $\xi_{(t)}^{v}=(1,0,0,0)$ and $\xi_{(\phi)}^{v}=(0,0,0,1)$.

The conserved quantities (the specific energy $E$ and azimuthal angular momentum $L_{\mathrm{z}}$ (per unit mass)) corresponding to Eq.(8) are given by,

$$
E=\dot{t h}(r), \quad L_{\mathrm{z}}=\dot{\phi} r^{2} \sin ^{2} \theta,
$$

where the dot represents the differentiation over the affine parameter $\tau$. By using normalization condition $u^{v} u_{v}=-1$, where $u$ is the four velocity, we obtain

$$
\dot{r}^{2}=E^{2}-\left(1-\frac{2 M_{\Theta}}{r}+\frac{Q_{\Theta}^{2}}{r^{2}}\right)\left(1+\frac{L^{2}}{r^{2} \sin ^{2} \theta}\right) .
$$

At tuning points of particles moving from trajectories $\dot{r}=0$

$$
E^{2}=\left(1-\frac{2 M_{\Theta}}{r}+\frac{Q_{\Theta}^{2}}{r^{2}}\right)\left(1+\frac{L^{2}}{r^{2} \sin ^{2} \theta}\right) \equiv V_{e f f}(r),
$$

where $V_{\text {eff }}$ represents the effective potential. The ISCO for the NCRN BH in the equatorial plane $\left(\theta=\frac{\pi}{2}, \dot{\theta}=0\right)$ can be obtained solving $V_{e f f}=0$

$$
r_{0}^{4} M_{\Theta}^{\prime}+r_{0}^{3}\left(-M_{\Theta}-Q_{\Theta} Q_{\Theta}^{\prime}\right)+r_{0}^{2}\left(L^{2}+Q_{\Theta}^{2}+L^{2} M_{\Theta}^{\prime}\right)-r_{0}\left(3 L^{2} M_{\Theta}+L^{2} Q_{\Theta} Q_{\Theta}^{\prime}\right)=0
$$

where

$$
\begin{aligned}
& M_{\Theta}^{\prime}=-\frac{e^{-\frac{r_{0}^{2}}{4 \Theta}} M r_{0} \sqrt{\frac{r_{0}^{2}}{\Theta}}}{2 \Theta \sqrt{\pi}}, \\
& Q_{\Theta}^{\prime}=\frac{Q^{2}}{\pi}\left[\frac{e^{\frac{r_{0}^{2}}{2 \Theta}}}{\Theta^{3 / 2} \sqrt{\frac{r_{0}^{2}}{\Theta}}}-\frac{\sqrt{2} r_{0}^{2} e^{\frac{r_{0}^{2}}{2 \Theta}}}{\Theta^{3 / 2} \sqrt{\frac{r_{0}^{2}}{\Theta}}}+\sqrt{2} \sqrt{\frac{1}{\Theta}} \gamma\left(\frac{1}{2}, \frac{r_{0}^{2}}{4 \Theta}\right)-\frac{2 r_{0} e^{\frac{r_{0}^{2}}{2 \Theta}} \gamma\left(\frac{1}{2}, \frac{r_{0}^{2}}{4 \Theta}\right)}{\Theta \sqrt{\frac{r_{0}^{2}}{\Theta}}}-\frac{\gamma\left(\frac{1}{2}, \frac{r_{0}^{2}}{2 \Theta}\right)}{\sqrt{2 \Theta}}\right] .
\end{aligned}
$$

The corresponding energy and azimuthal angular momentum are

$$
L_{\mathrm{z} 0}=\sqrt{\frac{M_{\Theta} r_{0}^{3}-Q_{\Theta}^{2} r^{2}-r_{0}^{4} M_{\Theta}^{\prime}+r_{0}^{3} Q_{\Theta} Q_{\Theta}^{\prime}}{r^{2}-3 M_{\Theta} r_{0}+2 Q_{\Theta}^{2}+r_{0}^{2} M_{\Theta}^{\prime}-r_{0} Q_{\Theta} Q_{\Theta}^{\prime}}}, E_{0}^{2}=\frac{\left(r_{0}^{2}-2 M_{\Theta} r_{0}+Q_{\Theta}^{2}\right)^{2}}{r_{0}^{2}\left(r^{2}-3 M_{\Theta} r_{0}+2 Q_{\Theta}^{2}+r_{0}^{2} M_{\Theta}^{\prime}-r_{0} Q_{\Theta} Q_{\Theta}^{\prime}\right)} .
$$

Now we consider that initially the particle is orbiting in ISCO, collides with another particle coming from infinity (initially at rest). There are three possible outcomes for the particle motion as a result of this collision, i.e, either have bounded motion around $\mathrm{BH}$, captured by $\mathrm{BH}$, or escape to infinity. If there is a small change in energy as well as the angular momentum, then the path of the particle is slightly changed. On the other hand, the major change in the energy and angular momentum can cause the particle to deviate from its original path, as a result, it can escape to infinity or attracted towards the BH. After colliding with another particle, there will be have new integrals of motion. We consider a simple case when the initial radial velocity as well as azimuthal angular momentum do not change after the collision. Now angular momentum and energy at the equatorial plane takes the form [5]

$$
L^{2}=L_{\mathrm{z} 0}^{2}+r_{0}^{2} v_{\perp}^{2}, \quad E=\sqrt{E_{0}^{2}+v_{\perp}^{2} h\left(r_{0}\right)} .
$$

If $E \geq 1$, the particle will escape to infinity this yields

$$
\left|v_{\perp}\right| \geq \sqrt{\left(1-E_{0}\right) h\left(r_{0}\right)^{-1}} .
$$




\section{Analysis of Effective Potential}

12

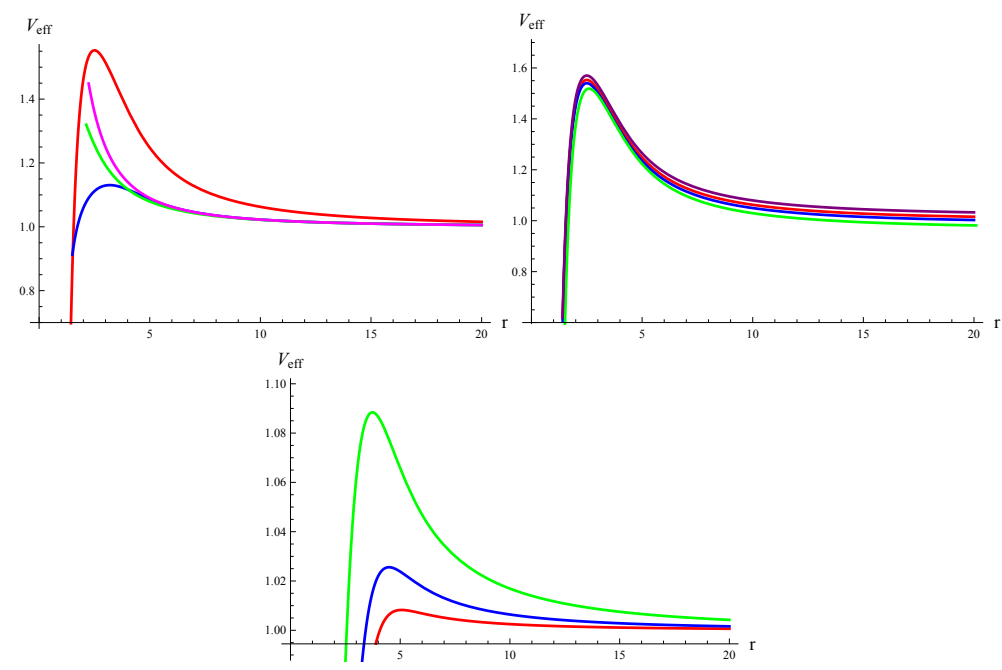

Figure 1. Effective potential with respect to $r$ for $M=1$ and $L=1.5$ (upper panel). In the left graph, $Q=0.5$ and red, blue, green, and pink curves corresponds to $\Theta=0.7,0.5,0.3,0$. In the right graph, $\Theta=0.5$ and red, blue, green, and purple curves corresponds to $Q=0.5,0.3,0.2,0$. In lower panel, $M=1, \Theta=0.5$ and $Q=0.5$. Here, red, blue, and green curves corresponds to $L=2.5,2,1.5$.

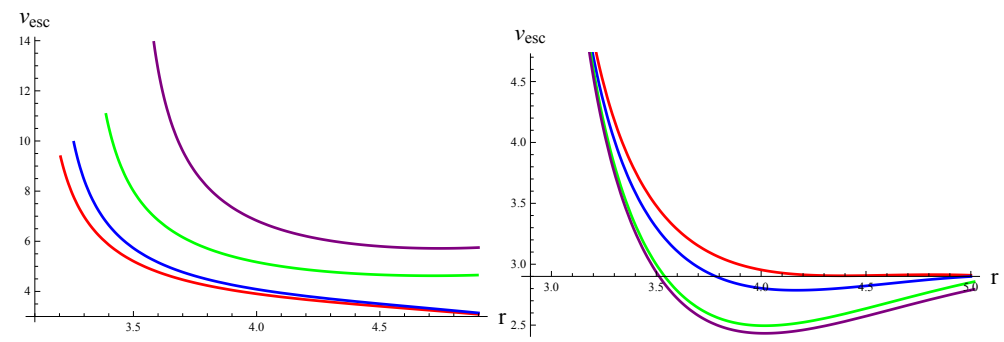

Figure 2. Escape velocity versus $r$ for $M=1$, and $L=1.5$. Here, red, blue, green, and purple curves corresponds to $\Theta=0.7,0.5,0.3,0$ (Left) and $Q=0.5,0.3,0.2,0$ (Right).

In this section, we examine the behavior of the effective potential as well as the escape velocity of the particle graphically using Eqs.(9) and (12). In Figure 1, the effective potential is plotted with respect to $r$. The stable and unstable orbits corresponds to the maximum and minimum values of the effective potential. In the left plot, we study the behavior of $V_{\text {eff }}$ by varying the values of $\Theta$. We observe that for the large values of $\Theta$, the effective potential attains higher values. Initially, the orbits are unstable but gradually $V_{e f f}$ decreases as $r$ increases which corresponds to stable orbits. The behavior of $V_{\text {eff }}$ for NCRN BH is similar to the case of RN BH. The right plot indicates $V_{\text {eff }}$ for the different values of $Q$. These plots indicate that the $V_{\text {eff }}$ has smaller values as $Q$ increases, also for 
the neutral $\mathrm{BH}(Q=0)$ the orbits are highly unstable. We also analyze the stability for the different values of the angular momentum. The graph in the lower panel describes that for the small values of angular momentum, the orbits are unstable while the large values of $V_{\text {eff }}$ correspond to stable paths.

In Figure 2, the escape velocity is plotted for the different values of $\Theta$ and $Q$. The left plot shows that the escape velocity is higher for $\Theta=0$, also the increasing values NC parameter correspond to less chances for the particle to escape from the vicinity of the $\mathrm{BH}$. The right plot indicates that the escape velocity of the particle becomes higher for the large values of charge while for $Q=0$, it is the smallest.

\section{Final Remarks}

In this paper we have investigated the particle dynamics orbiting around the NCRN BH. For this purpose, we first formulate the equations of motion. We have examine the behavior of effective potential as well as escape velocity of the particles moving around a charged NC BH. By observing the graphical properties of the effective potential, we found that the path of the particle is effected by the NC parameter. The largest value of NC parameter corresponds to highly unstable orbit, also the change in charge parameter (in the presence of $\Theta$ ) leads to both unstable and stable orbits. However, the orbits are more stable when the angular momentum is high. The cases of neutral and RN BHs have shown the similar behavior as compared to NCRN BH. We have also studied the effect of noncommutativity as well as charge of the $\mathrm{BH}$ on the particle's escape velocity. We observe that the particle have less chances to escape to infinity in the presence of $\mathrm{NC}$ parameter while the increasing values of the $\mathrm{BH}$ charge leads to high values of the escape velocity. We conclude that noncommutativity causes the deviation in the original path of the particle.

\section{References}

[1] P. Nicolini, Int. J. Mod. Phys. A 24, 1229 (2009).

[2] H. Garcia-Compean and C. Soto-Campos, Phys. Rev. D 74, 104028 (2006); K. Nozari and B. Fazlpour, Mod. Phys. Lett. A 22, 2917 (2007); R. Banerjee, B.R. Majhi and S. Samnta, Phys. Rev. D 77, 124035 (2008); Y.G. Miao, Z. Xue and S.J. Zhang, Int. J. Mod. Phys. D 21, 1250018 (2012); M. Sharif and S. Iftikhar, Eur. Phys. J. C 76, 630 (2016).

[3] V.P. Frolov and A.A. Shoom, Phys. Rev. D 82, 084034 (2010).

[4] V.P. Frolov, Phys. Rev. D 85, 024020 (2012).

[5] A.M. Al Zahrani, V.P. Frolov and A.A. Shoom, Phys. Rev. D 87, 084043 (2013).

[6] B. Majeed, S. Hussain and M. Jamil, Adv. High Energy Phys. 2015, 671259 (2015); M. Sharif, and S. Iftikhar, Int. J. Mod. Phys. D 26, 1750091 (2017).

[7] P. Nicolini, A. Smailagic and E. Spallucci, Phys. Lett. B 632, 547 (2006).

[8] S. Ansoldi, P. Nicolini, A. Smailagic and E. Spallucci, Phys. Lett. B 645, 261 (2007).

[9] P. Nicolini, J. Phys. A 38, L631 (2005).

[10] A. Smailagic and E. Spalluci, J. Phys. A 36, L517 (2003); J. Phys. A 37, 1 (2004).

[11] S.A., Alavi, Acta Phys. Polon. B 40, 2679 (2009). 